\title{
How do children with developmental language disorder extend novel nouns?
}

KRZEMIEN Magali ${ }^{\mathrm{a}, \mathrm{b}}$, THIBAUT Jean-Pierre ${ }^{\mathrm{c}}$, JEMEL Boutheina ${ }^{\mathrm{d}, \mathrm{e}}$, LEVAUX Emilie $^{\mathrm{a}}$, and MAILLART Christelle ${ }^{\mathrm{a}}$

${ }^{a}$ Department of Speech and Language Therapy, Research Unit on Childhood, University of Liège, B38, 30 rue de l'Aunaie, 4000 Liège, Belgium

${ }^{\mathrm{b}}$ FRESH fund, F.R.S.-FNRS

${ }^{\mathrm{c}}$ LEAD CNRS UMR 5022, University of Bourgogne Franche-Comté, Pôle AAFE, Dijon, France

${ }^{\mathrm{d}}$ Laboratoire de Recherche en Neurosciences et Électrophysiologie Cognitive, CIUSS_NIM, Montréal, QC, Canada

e École d'Orthophonie et d'Audiologie, Faculté de Médecine, Université de Montréal, Montréal, QC, Canada

Corresponding author: KRZEMIEN Magali, mkrzemien@alumni.uliege.be

\section{Highlights}

- Children with DLD are impaired in extending substance names and relational nouns.

- Vocabulary has an impact on novel noun extension.

- Comparison is beneficial for new word extension, especially in children with DLD.

- Children with DLD rely on shape when extending new nouns.

\begin{abstract}
In this study, we investigated the ability of children with developmental language disorder (DLD) to extend nouns referring to different categories of novel objects. In a word extension task, we used several types of object entities (solid, animate, non-solid, functional and spatial relations) for which children had to attend to diverse properties (either shape, or texture, or role,
\end{abstract}


or spatial relation) to decide category membership. We compared 15 school-aged children with DLD to typically developing (TD) children matched on either age or vocabulary. Our results indicate that children with DLD were impaired extending novel words for non-solid substances and relational objects, whereas age-matched TD children performed well for all object classes. Similar to children with DLD, TD children matched on language had difficulty extending spatial relation categories. We also show that children with DLD needed more learning exemplars and relied more on shape-based information than TD children did, especially for spatial configuration objects. Overall, our findings suggest that children are able to learn regularities between object properties and category organization, and so to focus on diverse features according to the object presented when extending novel nouns. They also provide clear evidence linking DLD to deficits in novel name generalization and word learning.

Keywords: developmental language disorder; generalization; vocabulary; comparison

\section{Introduction}

Vocabulary is the set of words of a language known by an individual. While vocabulary size grows quickly in typically developing children (Bloom, 2002), children with developmental language disorder (DLD) seem to struggle to expand their lexicon. DLD is a neurodevelopmental disorder characterized by impairments in language development and learning, including difficulties in novel word learning, and it is not due to a biomedical condition linked to genetic or neurological causes (Bishop et al., 2017). These language impairments persist until middle school and beyond, and they have an arguably deleterious impact on the child's everyday life.

Previous research has shown that children with DLD produce their first words later than their peers (Trauner, Wulfeck, Tallal, \& Hesselink, 2000). Furthermore, as reported by McGregor, Oleson, Bahnsen, and Duff (2013), their vocabulary is characterized by limited 
breadth (i.e., the number of words they know), but also depth (i.e., how well they comprehend the meaning of the words they know). In a word definition task (see McGrego et al., 2013), the authors showed that children with DLD produce significantly less informative definitions than typically developing (TD) children do, even when the analyses included only children who manifested a real ability to provide definitions. These findings suggest that children with DLD may learn novel words but the meaning of words remains less detailed than in TD children.

Word learning is a complex multidimensional process that encompasses several components such as phonological encoding, form-meaning mapping, lexical access, word retention and semantic knowledge and organization, as well as working memory (Kan \& Windsor, 2010, p. 740). Several studies have reported that children with DLD perform worse than their age-matched peers but similar to their language-matched peers in learning novel words (cf. for a review, Kan \& Windsor, 2010). In addition, they are slow to learn new words, have difficulty retaining new word labels, encode fewer semantic features of newly learned items, and require more exposure to novel words in order to learn them (Alt, Plante, \& Creusere, 2004; Jackson, Leitao, \& Claessen, 2016; Nation, 2013). It should be noted that these studies have focused mainly on the initial stage of lexical learning (form-meaning mapping) and not on all aspects of lexical development (Kan and Windsor, 2010). The authors considered broad variables such as the number of novel word exposures, the types of words (e.g., nouns or verbs), types of tasks or output formats (e.g., identification or production of the words) or the presentation format (e.g., fast or slow). These factors tell us what children learned easily (e.g. nouns better than verbs) or the best way to discriminate children with or without DLD (e.g. testing after high exposure or, with comprehension and recognition tasks), but nothing about the underlying causes of these difficulties.

A promising line of research aiming at understanding word learning difficulties in children with DLD has been to investigate the cognitive processes known to support typical 
lexical acquisition, such as novel word generalization (i.e. word extension). When young children learn a novel word, they have to understand the set (category) of instances to which this word refers to be able to extend or generalize it to new instances in various contexts (Gentner \& Namy, 1999; Kan \& Windsor, 2010). For example, if a child encounters an animal and he/she is told that it is called "poodle", the child must understand the features characterizing "poodles" in order to extend it accurately later, while restricting the word "poodle" to poodles only. Furthermore, novel name extensions are not constrained in the same way across noun types. It has been argued that children learn very early that names for solid objects apply to objects that share the same shape. To illustrate, when children learn the word "cup," they have to specifically attend to the object shape because shape is a relevant property when deciding whether or not an object can be included in the "cup" category (Smith, Jones, Landau, Gershkoff-Stowe, \& Samuelson, 2002). This shape bias favors shape over other properties, such as size or color (Gershkoff-Stowe \& Smith, 2004; Jones \& Smith, 1998). The shape bias has been consistently observed in 2- or 3-year-old children (Gershkoff-Stowe \& Smith, 2004; Jones \& Smith, 1998; Samuelson \& Smith, 2000). Smith et al. (2002) have shown that teaching 17month-old infants nouns for categories well organized by shape resulted in these children developing a shape bias for these categories, but also for other novel solid object categories. Interestingly, infants who developed the shape bias increased their vocabulary for solid objects outside the laboratory during the course of the study. This bias is, therefore, an important tool for learning in young children.

Other categories are organized by perceptual features other than shape and other word extension biases are at play. For example, when objects are identified as animates, the shape bias is weakened and texture becomes a critical feature to extend new words. Jones, Smith, and Landau (1991) showed that 2- and 3-year-old children extended new words by shape when objects had no eyes but by shape and texture when eyes were added to the same objects. Jones 
and Smith (1998) found that 3-year-olds extended novel nouns by shape for objects without shoes and by texture for objects with shoes. In this context, children beyond 2 years of age extend non-solid object names by texture and not by shape (Soja, Carey, \& Spelke, 1991). However, such a texture bias seems to be more fragile (Samuelson \& Horst, 2007) or more context dependent (Perry, Samuelson, Burdinie, 2014) than the shape bias.

Another category of nouns, relational nouns, has also received much attention. One feature of relational nouns is that they are not defined by perceptual features, but rather by extrinsic properties. "Their meanings include relations between other concepts" (Gentner, 2005, p. 248). Nouns such as predator, robbery, and neighbor are relational because they refer to a relational structure between entities (e.g., between two animals or between an individual and an object), while their referents are often visually dissimilar or have no common intrinsic property (Gentner, 2005). It is worth noting that relational nouns are acquired later than solid object names. In fact, there are few relational nouns in the Mac Arthur Communicative Developmental Inventory until 30 months of age (Gentner, 2005). Moreover, when relational nouns are acquired, they are often understood as object names defined by concrete features. Contrary to school-age children, preschoolers tend to define relational nouns in terms of objects perceptual properties. For example, a child finds it hard to believe that a 2-year-old infant could be an uncle because uncles have to be about 24 or 25 years old (Keil \& Batterman, 1984).

Regarding novel word learning, it has been argued that children are able to use different kinds of properties to extend novel nouns referring to various object classes, depending on the object they are confronted with (Jones \& Smith, 1998; Jones et al., 1991; Snape \& Krott, 2018). Thus, children seem to be sensitive to regularities between object properties and category organization. This sensitivity is important because of its impact on the size of the lexicon. For example, Thom and Sandhofer (2009) found that 20-month-old infants who were trained with more color words were better able to extend new color words than children who were trained 
with fewer words suggesting that word extension is facilitated in domains for which children have a labeling experience. Thus, as children learn more words, they develop attentional biases towards properties that are relevant to category membership (Jones \& Smith, 1998). As object names are the most frequent in young children's daily life, it is not surprising that the shape bias is their first and most robust attentional bias (Samuelson, Horst, Schutte, \& Dobbertin, 2008; see Bloom, 2002, for a different interpretation, however).

Most of the evidence regarding the above attentional biases has been obtained with a single training exemplar design, in which participants are shown a training stimulus and, then, are asked to select among a set of options the one that has the same name. However, in many learning situations, two or more stimuli are introduced simultaneously with the same word, which gives children the opportunity to compare these stimuli before they generalize their name to novel instances. There is now ample evidence that a comparison format leads to more conceptually-based generalizations in novel noun learning tasks than single stimulus formats (see Augier \& Thibaut, 2013; Namy \& Gentner, 2002). A general explanation is that comparisons lead participants to first focus on salient similarities between training exemplars (e.g., shape) and, later on, search for deeper commonalities, even though less salient (Namy \& Gentner, 2002). This is especially the case for relational nouns, for which comparisons support the identification of relational over perceptual features in young children (Christie \& Gentner, 2010; Gentner, Anggoro, \& Kilbanoff, 2011; Thibaut \& Witt, 2015, 2017). For example, Christie and Gentner (2010) found that children 3 and 4 years old were able to extend a novel noun for a spatial relation when two learning exemplars were provided, but not when only one learning exemplar was presented. Multiple object presentation favors generalization in word extension tasks, although, it can give rise to generalizations based on salient but irrelevant properties (see Augier \& Thibaut, 2013).

\section{Novel noun extension in children with DLD.}


Only a few studies have systematically investigated novel noun extension in children with DLD. Schwartz, Leonard, Messick, and Chapman (1987) found that these children apply a new noun to unnamed exemplars less than their language-matched peers. Gray (1998) also found that children with DLD do not perform as well as age-matched peers when they had to extend words to new contexts (i.e., color photographs or black line drawings). More recently, Collisson, Grela, Spaulding, Rueckl, and Magnuson (2015) found that contrary to their agematched peers, preschool-age children with DLD did not exhibit a shape bias. They did not preferentially extend solid object names by shape, since they relied equally on shape, color, and texture. DLD, therefore, seems to be associated with a word extension impairment, in addition to deficits in other aspects of word learning, such as semantic representation or form-meaning mapping (Kan \& Windsor, 2010; McGregor et al., 2013).

However, while other classes of nouns have received much attention in TD children, no studies have investigated word extension for words other than solid object names in children with DLD, to the best of our knowledge. Pandolfe, Wittke, and Spaulding (2016) have shown that adolescents with DLD were impaired in their knowledge of words related to driving, which are abstract and, for many of them, relational words. Moreover, previous studies with children with DLD have shown that learning other types of relational words, such as verbs, is problematic for this population (Windfuhr, Faragher, \& Conti-Ramsden, 2002). This is interesting because the difficulties of children with DLD in learning verbs suggest that learning other types of words such as relational nouns may also be hampered in this population.

Whilst being exposed to two learning instances facilitates relational noun extension in TD children (Christie \& Gentner, 2010; Gentner et al., 2011), such an experimental setup might not benefit children with DLD since DLD is associated with difficulty in using comparisons and structural alignment (Leroy, Maillart, \& Parisse, 2014; Leroy, Parisse, \& Maillart, 2012). These children also have difficulty identifying relational similarities when they are not 
supported by perceptual features (Leroy et al., 2012, 2014). In analogical reasoning tasks, Leroy et al. (2014) showed that children with DLD had poorer performance in a linguistic task (composed of syllables) and a non-linguistic analogical reasoning task (composed of pictures) in which they had to complete a sequence sharing the same relational structure as previously presented sequences. Interestingly, in the linguistic task, the performance of children with DLD was poorer for relational items that were not backed up by perceptual cues (e.g., /na-ba-ba/ and /ty-sy-sy/) than relational items that were backed up by perceptual cues (e.g., /my-ly-ly/ and /me-le-le/). This suggests that relational terms themselves may be difficult to learn and generalize by children with DLD when different instances of a relation do not share any perceptual cue (e.g., different classes of objects can be connected by the "neighbor of" relational noun).

In sum, children with DLD have difficulty learning and extending new words (Gray, 1998; Kan \& Windsor, 2010; Schwartz et al., 1987). They do not develop a shape bias at the same age as their TD peers (Collisson et al., 2015) and they have difficulty learning abstract and relational words (Pandolfe et al., 2016; Windfuhr et al., 2002). Moreover, children with DLD are impaired in using comparison, and they have difficulty focusing on relational rather than perceptual features (Leroy et al., 2012, 2014). In contrast, TD children can identify regularities across objects in order to extend novel names for different classes of entities. TD children also develop attentional biases towards features that are relevant to category membership (Jones \& Smith, 1998; Jones et al., 1991; Soja et al., 1991). In this context, withincategory comparisons seem to contribute to the abstraction of conceptually relevant properties, especially for words that are not defined by perceptually-based features, such as relational nouns (Christie \& Gentner, 2010; Gentner et al., 2011; Thibaut \& Witt, 2015).

\section{Present study}


The main purpose of this study was to examine generalization abilities in school-age children with DLD using a word extension task. During the experiment, children were first introduced to an unfamiliar object together with its name (a non-word) and, then they were asked to indicate the other stimulus to which they would generalize the novel noun.

This study has four objectives:

First, we evaluate the ability of children with DLD to extend nouns associated with different kinds of entities and to identify the relevant properties to category membership according to the object that has been introduced. Indeed, novel noun generalization abilities have not been compared for nouns referring to various types of entities. Thus, comparing how novel words for various types of entities are acquired remains an open question. We compared extensions of novel nouns for five object classes (solid objects, animate objects, non-solid substances, functional role categories, and spatial configuration categories). These object classes have already been examined separately (Christie \& Gentner, 2010; Gentner et al., 2011; Jones \& Smith, 1998; Smith et al., 2002; Soja et al., 1991) or in comparison (e.g. solid and nonsolid objects, see Kucker et al., 2019 for a recent review) in various studies in TD children, but - to our knowledge - these five classes have never been compared in the same study in children with DLD or in TD children.

Second, we have seen that these abilities are related to the size of the lexicon (Smith et al., 2002; Thom \& Sandhofer, 2009). It is, therefore, possible that children with DLD are impaired relative to age-matched peers but they perform similarly to children matched on a vocabulary measure. We thus include two TD matched-groups, one younger (the languagematched group) and one older (the age-matched group). This double-matching procedure will allow us to disentangle language level issues from age-related issues.

Third, we will contrast single and multiple object presentations. Indeed, it has been repeatedly shown that the opportunity to compare multiple instances of the same category leads 
to more accurate generalizations (Christie \& Gentner, 2010; Gentner \& Namy, 1999). Given the limited size of our available sample of participants with DLD, we could not run the comparison and the single conditions as a between-subject factor. Thus, we decided to first start with a single occurrence, and, in case of failure, multiple exemplars of the same category were presented. Note that this new methodological within-subject approach corresponds to what may happen in real-world situations. Children sometimes encounter one stimulus of a category and later two (or more) specimens of the same category.

Fourth, we analyze a possible generalization bias of children with DLD to focus on salient perceptual features, even in relational categories. Indeed, as mentioned above, younger children sometimes generalize novel relational nouns based on perceptual similarities (Christie \& Gentner, 2010; Keil \& Batterman, 1984). This may also happen with children with language deficits, as these children have already been found to favor perceptual similarities over relational features (Leroy et al., 2012, 2014).

\section{Methods}

\section{Participants}

Forty-nine children were recruited for this study; 19 were identified as having DLD and 30 were TD children. Children were recruited in the French-speaking region of Belgium. Informed consents were obtained from parents through schools, as well as information about children's medical and developmental history. Children were recruited if parents did not report any hearing impairment or neurological disorder. As bilingualism could have impacted language assessment, all children were monolinguals. The study received the approval of the local ethics committee.

Children with DLD, aged between 7;3 to $12 ; 4$ years old, were recruited through schools for children with special needs. All children with DLD had been diagnosed before their enrollment in this study. We performed a language assessment protocol that confirmed their 
current diagnosis. Receptive abilities were assessed with a picture-pointing task involving words for vocabulary (Evaluation du Vocabulaire en Images Peabody, EVIP, Dunn, ThériaultWhalen, \& Dunn, 1993) and a picture-pointing task involving sentences for the morphosyntax domain (Epreuve de COmpréhension Syntaxico-SEmantique, ECOSSE, Lecocq, 1996, French adaptation of the TROG). Expressive abilities were evaluated with the ELO battery (Evaluation du Langage Oral, Khomsi, 2001), which includes a word repetition task in phonology, a picturenaming task in vocabulary, and a sentence completion task in morphosyntax. Children were selected if they scored below the $10^{\text {th }}$ percentile in at least two language components and, thus, matched Leonard's criteria for specific language impairment (i.e., the previous designation of DLD, Leonard, 2014). The most frequently impaired language components were phonology and morphosyntax. Finally, children all had a nonverbal intellectual quotient (NVIQ) of at least 80 (Echelle non verbale d'intelligence de Wechsler, Wechsler \& Naglieri, 2009).

Table 1

Characteristics of the age-matched groups

\begin{tabular}{|c|c|c|c|}
\hline & $\begin{array}{c}\text { Children } \\
\text { with DLD } \\
(\mathbf{n}=15)\end{array}$ & $\begin{array}{l}\text { Age-matched } \\
\text { children } \\
(n=15)\end{array}$ & \\
\hline Gender & $\begin{array}{l}7 \text { girls; } 8 \\
\text { boys }\end{array}$ & 9 girls; 6 boys & $X^{2}(1)=0.13$ \\
\hline $\begin{array}{l}\text { Age } \\
\text { Year;Month }(S D)\end{array}$ & $10 ; 0(1 ; 6)$ & $10 ; 1(1 ; 7)$ & $t(28)=0.18$ \\
\hline $\begin{array}{l}\text { NVIQ } \\
\text { Mean }(S D)\end{array}$ & $96.5(9.8)$ & $97.1(10.5)$ & $t(28)=0.16$ \\
\hline $\begin{array}{l}\text { Word repetition } \\
\text { Raw score - Mean (SD) }\end{array}$ & $24.3(7.3)$ & $31.9(0.3)$ & $W=225.00 * * *$ \\
\hline $\begin{array}{l}\text { Picture-pointing task (word) } \\
\text { Raw score - Mean (SD) }\end{array}$ & $90.9(16.5)$ & $117.0(12.6)$ & $t(28)=4.87 * * *$ \\
\hline $\begin{array}{l}\text { Picture-naming task } \\
\text { Raw score - Mean (SD) }\end{array}$ & $29.5(3.4)$ & $35.2(2.6)$ & $t(28)=5.15^{* * *}$ \\
\hline $\begin{array}{l}\text { Picture-pointing task (sentence) } \\
\text { Error score - Mean (SD) }\end{array}$ & $12.4(4.3)$ & $6.08(3.0)$ & $t(28)=-4.64 * * *$ \\
\hline
\end{tabular}




\section{Sentence completion}

Raw score - Mean (SD)
$13.9(3.3)$
$W=213.00 * * *$

Note. $\mathrm{SD}=$ standard deviation, $\mathrm{NVIQ}=$ non-verbal intellectual quotient, $* p<.05, * * * p<.001$.

Regarding TD children $(N=30)$, none had repeated a year in school and none had a history of language or other developmental disorder. We conducted the same language evaluation protocol with TD children; they all performed above the $10^{\text {th }}$ percentile in every language component. Fifteen TD children (age range $=7-11 ; 11$ years old) were individually matched with children with DLD on chronological age ( \pm 6 months) and on NVIQ ( \pm 8 points). However, four children with DLD could not be matched to a TD child due to low NVIQ scores. Overall, TD and DLD age-matched groups did not differ in age, gender, and NVIQ, but differed in all language measures (Table 1). Fifteen additional TD children (age range $=5 ; 9-10 ; 11$ years old) were individually matched to children with DLD according to a vocabulary comprehension measure ( \pm 8 points, EVIP, Dunn et al., 1993). A vocabulary comprehension measure was chosen because vocabulary has been related to the ability to extend new words (Jones \& Smith, 1998; Thom \& Sandhofer, 2009). Four children with DLD could not be matched to TD children due to low scores on the vocabulary measure. The group of children with DLD and their TD language-matched group did not differ in gender, NVIQ, vocabulary comprehension, and sentence comprehension, but they differed in age and all other language measures (Table 2).

Table 2

Characteristics of the language-matched groups

\begin{tabular}{lccc}
\hline & $\begin{array}{c}\text { Children with } \\
\text { DLD } \\
(\mathbf{n}=\mathbf{1 5})\end{array}$ & $\begin{array}{c}\text { Language- } \\
\text { matched children } \\
(\mathbf{n}=\mathbf{1 5})\end{array}$ & \\
\cline { 2 - 3 } Gender & 9 girls; 6 boys & 9 girls; 6 boys & $X^{2}(1)=0.00$ \\
$\begin{array}{l}\text { Age } \\
\text { Year;Month }(S D)\end{array}$ & $10 ; 5(1 ; 7)$ & $8 ; 1(1 ; 7)$ & $t(28)=-3.88^{* * * *}$
\end{tabular}




\begin{tabular}{lccc}
\hline $\begin{array}{l}\text { NVIQ } \\
\text { Mean (SD) }\end{array}$ & $96.8(12.4)$ & $100.2(12.9)$ & $t(28)=0.74$ \\
$\begin{array}{l}\text { Word repetition } \\
\text { Raw score - Mean }(S D)\end{array}$ & $24.2(8.5)$ & $31.3(1.1)$ & $W=210.00^{* * *}$ \\
$\begin{array}{l}\text { Picture-pointing task (word) } \\
\text { Raw score - Mean }(S D)\end{array}$ & $94.6(15.9)$ & $95.6(17.6)$ & $t(28)=0.16$ \\
$\begin{array}{l}\text { Picture-naming task } \\
\text { Raw score - Mean (SD) }\end{array}$ & $29.9(3.8)$ & $33.6(4.3)$ & $W=185.00 * *$ \\
$\begin{array}{l}\text { Picture-pointing task (sentence) } \\
\text { Error score - Mean (SD) }\end{array}$ & $12.3(5.2)$ & $9.2(4.2)$ & $t(28)=-1.78$ \\
$\begin{array}{l}\text { Sentence completion } \\
\text { Raw score - Mean }(S D)\end{array}$ & $14.1(3.6)$ & $18.8(3.9)$ & $t(28)=3.42 * *$ \\
$\begin{array}{l}\text { Note. SD= standard deviation, NVIQ= non-verbal intellectual quotient, } * p<.05, * * p<.01, \\
* * * p<.001 .\end{array}$ & &
\end{tabular}

\section{Materials}

This study aimed at evaluating the abilities of children with DLD and TD children to extend novel names to other instances of novel categories of objects. We used the word extension task paradigm, in which children are presented with a non-word such as "padi" associated with an object (Gentner \& Namy, 1999). We constructed thirty non-words that followed the consonant-vowel-consonant-vowel (CV-CV) structure typical of the French language (see appendix). All the syllables had a phonotactic frequency higher than 1000 (according to Manulex database, Lété, Sprenger-Charolles, \& Colé, 2004).

Visual stimuli consisted of colored pictures presented on a white background. Pictures depicted different objects belonging to one of five categories of objects (solid, non-solid, animate, spatial configuration and functional relational objects). As detailed below, category membership was determined by specific critical properties.

i) Solid objects referred to a category of shape-based objects. As mentioned above, solid objects have been related to a shape bias in TD children, which means that children should attend to shape when generalizing solid object names (Gershkoff-Stowe \& Smith, 2004; Smith et al., 2002). Five different sets of solid objects were created. Each set comprised six objects 
that shared similar shapes but had different textures and colors (see Figure 1). Two types of distractor stimuli were used, one included pictures of novel objects that resembled the learning exemplar in color only (e.g. color match) and the other one included pictures of novel objects that resembled the learning exemplar in texture only (e.g. texture match).

ii) Non-solid objects were textured shapes designed to illustrate existing substances, such as cream, water, or jam (Perry, Samuelson, Malloy, \& Schiffer, 2010; Soja et al., 1991). Five sets of non-solid objects were created. Each set comprised six objects that shared similar textures but had different shapes and colors (see Figure 1). Two types of distractor stimuli were selected, one included pictures of novel objects that resembled the learning exemplar in shape only (e.g. shape match) and the other one included pictures of novel objects that resembled the learning exemplar in color only (e.g. color match).

iii) We also used a category of animate objects. Animate objects were similar to solid objects, except that they had eyes. Eyes have been consistently regarded as being a cue for animacy (Jones et al., 1991) and animacy has been shown to draw children's attention to shape and texture (Jones \& Smith, 1998; Jones et al., 1991). Five sets of animate objects were created. Each set comprised six objects that shared similar shapes and textures but had different colors (see Figure 1). Two types of distractor stimuli were assembled, one included pictures of novel animate objects whose shape and color matched the learning exemplar's (e.g. shape \& color match) and the other one whose color and texture matched the learning exemplar's (e.g. color $\&$ texture match).

iv) The fourth category of objects used in our experiment referred to the spatial configuration category. For these objects, category membership relied on the relational pattern of its constituent parts. As shown in Figure 1, the learning exemplar and the target stimulus shared the same spatial configuration as they depict an object composed of a small element placed on top of a bigger element. Given that spatial configuration is not relevant to object 
categorization in the real world, children cannot use their knowledge of existing categories to learn the rule underlying object categorization for such category of objects. Moreover, Christie and Gentner (2010) found that preschool-aged children extended a novel noun for a spatial relation when they were presented with two learning exemplars. The spatial configuration is a property that children can learn to decide category membership, albeit through within-category comparison. Learning the driving rule for this category of objects would require that participants be exposed to more experimental materials, and thus more trials. Therefore, we created ten different sets of spatial configuration objects. Each set comprised six objects that shared similar spatial relational configurations but had different shapes and colors. Two types of distractor stimuli were selected, one included pictures of novel objects that resembled the learning exemplars in shape only (e.g. shape match) and the other one included pictures of novel objects that resembled the learning exemplars in color only (e.g. color match).

\footnotetext{
Figure 1

Examples of stimuli used for each category of objects: i) solid, ii) non-solid, iii) animate, iv) spatial configuration, and v) functional role. For each category of objects, the first and the second rows display respectively a learning exemplar and the corresponding generalization target object, and the two last rows show related distractor stimuli (distractor 1 and distractor 2).
} 


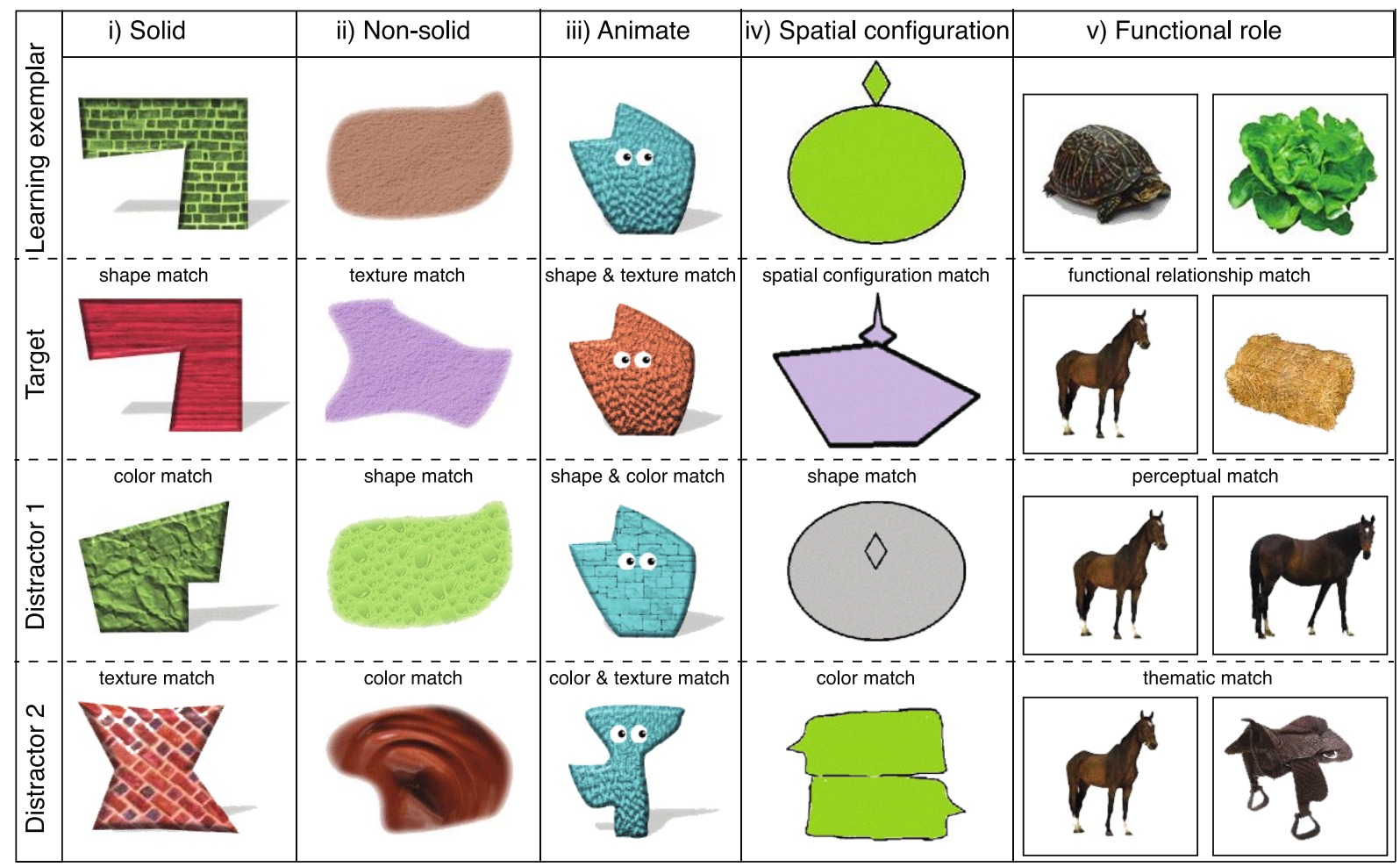

v) Finally, following Thibaut and Witt's study (2015), we also used a category of objects defined by functional relationship. For this latter category, all stimuli were photographs of real objects, such as "horse", "duck", "pencil", etc. (see Figure 1). To illustrate category membership, stimuli were always presented in pairs. In fact, presenting two objects side by side better highlighted the specific functional role that describes the nature of the relationship between these two objects. Five sets of pairs of real objects were created. Each set comprised six pairs of objects that shared similar functional relational role but had different object entities. As shown in Figure 1, the learning exemplar and the target stimulus of the functional role category shared the same thematic relationship, i.e., the turtle eats the lettuce as the horse eats the hay. Two types of distractor stimuli were assembled, one included a pair of objects whose relationship was perceptually based (i.e., two different horses) and the other pair displaying a thematic relationship that is different from the one depicted by the learning exemplar (i.e., horse and its saddle). 


\section{Procedure}

Children were tested individually in a quiet room at their home or at their school, during a session of about $30 \mathrm{~min}$. The experiment was run on a PC laptop computer using OpenSesame software (Mathôt, Schreij, \& Theeuwes, 2012). The task was introduced to each child in an entertaining context in which a friendly alien asked the child to learn words of his language.

The experimental session begun with two familiarization trials during which children were shown a novel object associated with a novel word. In the first trial, a learning exemplar of a category of objects appeared at the center of the upper half of the screen monitor as the experimenter labelled it with a non-word "You see this object, this is a dufan in the alien language". Three pictures of test objects, or response stimuli, were then presented below the learning exemplar. One of the response stimuli belonged to the same category as the learning exemplar, i.e. target object, and the other two were distractors. The experimenter then asked "Now, can you show me another dufan among these objects?". The children were instructed to indicate their choice by pressing one of three keyboard buttons. The spatial arrangement of the three response buttons mapped the spatial position of the three response stimuli on the screen, i.e. left, center, right. The second trial proceeded in a similar fashion but exhibited two learning exemplars rather than one exemplar belonging to the same category. The correct response was never provided (to facilitate the reading, we use correct response for the expected response given the attentional biases observed in previous studies - see Materials section - while incorrect response refers to the distractors).

After the familiarization phase, children received five experimental blocks. In each block, they were exposed to one of the five categories of object (solid, non-solid, animate, spatial configuration and functional relational objects). The order of presentation of these sets was counterbalanced across participants, except for the spatial relation category which was always preformed as the last experimental block. Given that spatial configuration is not a common 
criterion for object categorization (Christie \& Gentner, 2010), we speculated that children's understanding of category relationship for this category of objects may differ from that of the other categories of objects used in this study that would rely on more common object properties (shape, texture, thematic relations).

In each block, except the spatial relation category block, children were exposed to five different non-words associated with a specific target object category. The non-word labels were randomly assigned to target objects. For the spatial relation category block, children were exposed to ten different non-words. In order to evaluate the facilitatory effect of within-category comparison on new word extension (Christie \& Gentner, 2010; Gentner et al., 2011; Thibaut \& Witt, 2015), every novel noun was presented with one learning exemplar. In case of failure in this first trial, the same noun was introduced with two learning exemplars. If the child failed again, the same noun was presented with three learning exemplars. This means that the number of attempts to identify the correct object among the response stimuli could vary across children, being five at its minimum and 15 at its maximum. For the spatial relation block, the number of attempts ranged between 10 and 30 trials.

Figure 2 illustrates the unfolding of a test trial for the experimental blocks involving solid, non-solid, animate and spatial configuration objects (Figure 2A) and for the experimental block involving the functional role category of objects (Figure 2B). As shown in figure 2A, the test trial for each non-word started with the presentation of one learning exemplar of one specific category of objects: the experimenter pointed to the object's picture and labelled it by saying to the participant, "This is the padi. Look carefully at the padi." All non-words have been introduced by saying "This is the ..." rather than "This is $a$..." (Imai \& Gentner, 1997). While the learning exemplar remained on the screen, three different pictures of objects (the target object/correct response and two distractors/incorrect responses) appeared below the learning exemplar; and the experimenter said: "Show me the other padi". The position of the individual 
response stimuli (left, middle, or center) were randomly determined. In case where the child chose the target object, that is the correct response, the subsequent trial involved learning another non-word associated to another object of the same object category. In case of failure (see Figure 2A), no feedback was provided, but the same non-word was presented again with two learning exemplars, the same learning exemplar as in the previous trial and another one (see Figure 2A). The experimenter said: "This is the padi and this is also the padi" and added "Look at the reason why they are both padi". Some authors have indeed found that comparison must be explicitly encouraged to be beneficial in word extension tasks (Christie \& Gentner, 2010). Then, children were presented with three response stimuli and were asked to show "the other padi". If the child failed to make the correct choice, the same non-word was again presented with three learning exemplars, and the procedure was repeated. If the child failed again, no other exemplar was presented for this non-word and the next trial moved to the presentation of another non-word which began with no feedback other than neutral encouragement. Finally, after every object class, the child was given a reward to encourage motivation.

As depicted in figure $2 \mathrm{~B}$, test trials for the functional relation categories were slightly different from the other blocks. The test trials started with the presentation of a pair of real objects (e.g., a turtle and a lettuce), the experimenter said: "This is the cori of the turtle" while pointing to the two pictures. Then, the child was presented with a picture of a horse and had to select "the cori of the horse" among three possibilities. This syntactic structure has been found to orient 4-year old children to extending new words by role, even with only one learning exemplar (Gentner et al., 2011). Thus, children should select the object that has the same role as the learning exemplar (Gentner et al., 2011; Thibaut \& Witt, 2015).

Figure 2 
A. Schematic representation of a test trial involving non-word labels associated with a solid object. In case where the participant failed to choose the correct response (i.e., the target stimulus), the following trial presented the same non-word along with two learning exemplars, and the participant was asked again to find a similar object among the three response stimuli. B. Schematic representation of a test trial involving non-words associated with a functional role category of objects. In case where the participant chose the correct response stimulus, as illustrated here, the following trial presented another set of objects.

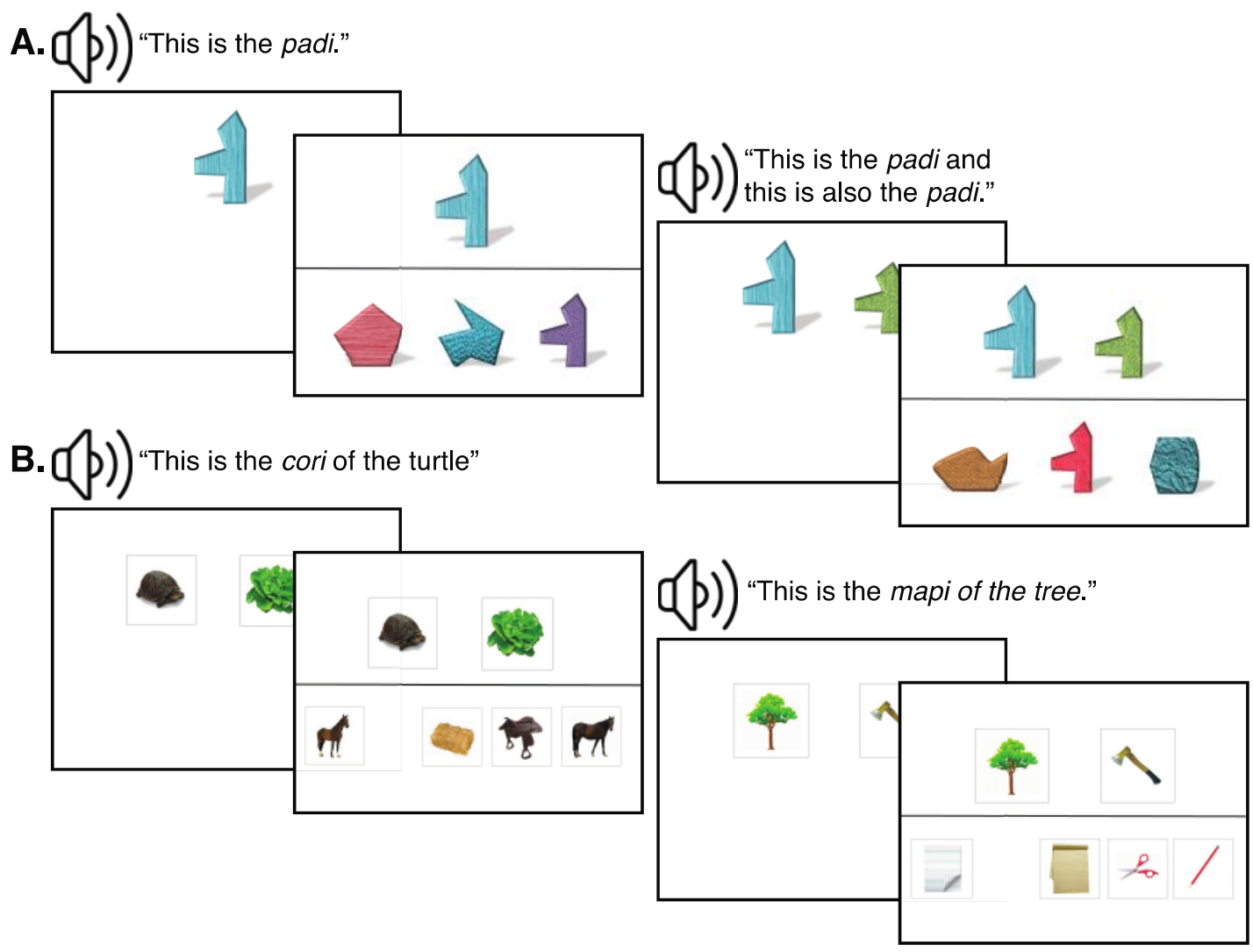

\section{Results}

First, we evaluated children's success rate at identifying the relevant property for category membership after being exposed to only one learning exemplar. Therefore, we conducted analyses of variance (ANOVAs) comparing the performance of a group of children with DLD to an age-matched group on the one hand, and to a language-matched group on the other hand, when one learning exemplar was provided, as a function of the object class (ezANOVA of the ez package in R, Lawrence, 2011). The scores of age-matched and language-matched groups were computed in two separate analyses, because a subset of children with DLD could not be matched either to a TD child on age (in the age-matched case) or on language (in the language- 
matched case). Thus, the two subgroups of children with DLD cannot be considered as one homogeneous group that would be equivalent to both control groups. Then, to further evaluate the impact of vocabulary on novel noun extension, we added the vocabulary measures to the models. We also verified that the percentages differed from chance level using one-sample t/Wilcoxon-tests. In a second analysis, we examined the impact of within-category comparisons (i.e., comparison trials) on novel word extension. We performed ANOVAs on the mean number of exemplars used by age-matched groups and language-matched groups to extend novel words, as a function of the object class. Thus, we examined the number of learning exemplars that children needed to successfully extend each novel noun, and we computed the mean for every object class. Finally, we investigated the role of the salient stimuli features by analyzing the type of distractor children selected in case of errors. We conducted ANOVAs to compare the number of incorrect responses as a function of the group and distractor for non-solid substances, functional role categories, and spatial configuration categories. The trials involving one, two, and three learning exemplars were computed together for these last analyses.

\section{Percentage of correct responses following one learning exemplar}

Age-matched groups

We conducted a $2 \times 5$ ANOVA on the percentage of correct responses in the one-learning exemplar case, with group (DLD vs. age-matched) as a between-subjects factor and category of objects (solid objects vs. animate objects vs. non-solid substances vs. functional role categories vs. spatial configuration categories) as a within-subjects factor. The main effect of group was not significant, $F(1,28)=3.21, p=.084, \eta_{\mathrm{p}}{ }^{2}=.10$. Moreover, the analysis yielded a significant effect of object category, $F(4,112)=9.90, p<.001, \eta_{\mathrm{p}}{ }^{2}=.26$, and more importantly a significant interaction between group and object category, $F(4,112)=7.45, p<.001, \eta_{\mathrm{p}}{ }^{2}=.21$. Post-hoc analyses (Tukey HSD) revealed that, while the two groups performed similarly on trials with solid and animate objects $(p>.10)$, age-matched children outperformed children 
with DLD on trials with non-solid substances $(p<.001)$, functional role categories $(p=.020)$, and spatial configuration categories $(p=.002)$. Furthermore, whilst the performance of agematched children did not differ significantly across the five categories of objects (all $p>.05$ ), children-with-DLD's performance varied significantly as a function of object category (see Figure 3A). Follow-up post-hoc analyses showed that children with DLD performed better on trials with solid and animate objects than on trials with non-solid substances and spatial configuration categories $(p<.001)$, on trials with solid objects than on trials with role categories $(p=.021)$. These results are presented in Figure 3A.

\section{Figure 3}

Mean percentages of correct responses following one learning exemplar are displayed as a function of the category of objects and as a function of the group (A. compares children with DLD and age-matched TD children, B. compares children with DLD and language-matched TD schildren)

A.

$\square$ Age-matched children

$\square$ Children with DLD

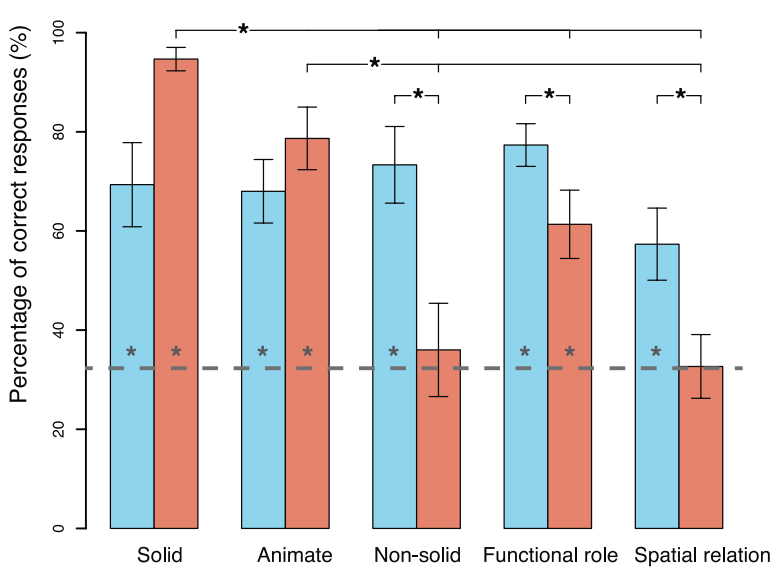

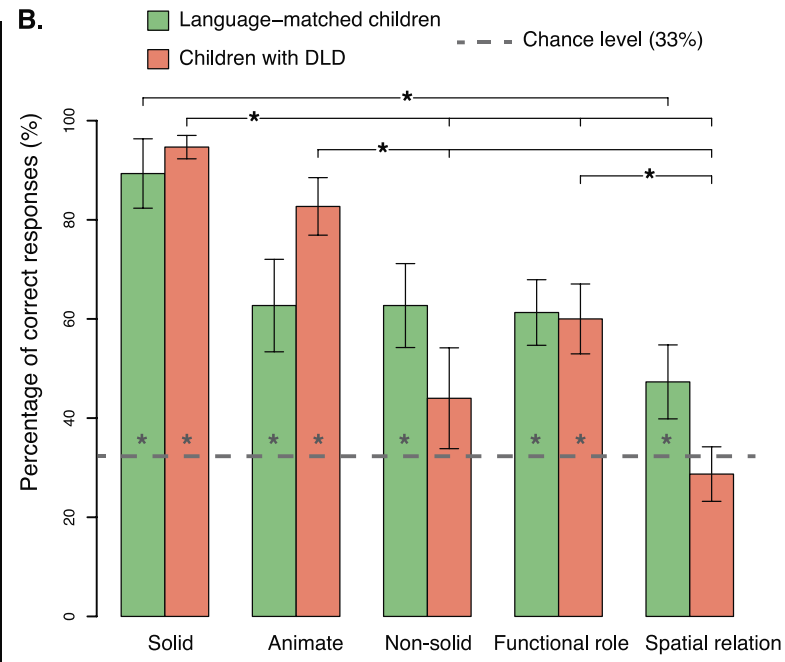

Note. Bars represent the standard error of the mean (SEM), $* p<.05$. The grey dashed line marks the chance level and the grey asterisk indicates significant above-chance performance.

To further investigate the relationships between vocabulary and noun extension, we added the vocabulary measures to the model. Therefore, we conducted an ANCOVA on the percentage of correct responses when one learning exemplar was provided, with group as a between-subjects factor, the object category as a within-subjects factor, and receptive and 
expressive vocabulary measures as covariates. The main effect of group was not significant, $F(1,26)=4.11, p=.053, \eta_{\mathrm{p}}^{2}=.14$. The effect of object category, $F(4,112)=9.90, p<.001$, $\eta_{\mathrm{p}}^{2}=.26$, and the interaction, $F(4,112)=7.45, p<.001, \eta_{\mathrm{p}}^{2}=.21$, still reached significance. Interestingly, the effect of receptive vocabulary was also significant, $F(1,26)=5.89, p=.022$, $\eta_{\mathrm{p}}^{2}=.18$, contrary to the effect of expressive vocabulary, $F(1,26)=3.90, p=.059, \eta_{\mathrm{p}}^{2}=.13$.

To assess whether the observed patterns of results were due to chance responding, we compared each group's performance in each object category condition to chance level (i.e., $33 \%)$. The analyses showed that age-matched children performed above chance level in all object category blocks (all $p<.01$ ). Children with DLD performed above chance level for solid objects, animate objects and functional role categories (all $p<.01$ ), but their performance was at chance level for non-solid substances, $t(14)=0.29, p=.78$, and spatial configuration categories, $t(14)=-0.098, p=.92$.

\section{Language-matched groups}

We conducted a $2 \times 5$ ANOVA on the percentage of correct responses when one learning exemplar was provided, with group (DLD vs. language-matched) as a between-subjects factor and object category (solid objects vs. animate objects vs. non-solid substances vs. functional role categories vs. spatial configuration categories) as a within-subjects factor. Data are reported in Figure 3B. The analysis did not reveal any group effect, $F(1,28)=0.22, p=.64, \eta_{\mathrm{p}}{ }^{2}=.003$. The effect of object category reached significance, $F(4,112)=18.1, p<.001, \eta_{\mathrm{p}}^{2}=.39$, and so did the interaction between group and object category, $F(4,112)=2.97, p=.022, \eta_{\mathrm{p}}^{2}=.096$. Post-hoc analyses using Tukey method revealed that children with DLD performed similarly to TD children for all object categories (all $p>.64$ ). The significant group by object category interaction indicated, however, that the performances of children-with-DLD varied more as a function of object category. Language-matched children performed better on trials with solid objects than with spatial configuration categories $(p=.001)$. In contrast, children with DLD 
performed better on trials with solid objects than with non-solid substances $(p<.001)$, functional role $(p=.016)$, and spatial configuration categories $(p<.001)$. They also performed better on trials with animate objects than with non-solid substances $(p=.004)$ and spatial configuration categories $(p<.001)$. Finally, they performed better on trials with functional role than with spatial configuration categories $(p=.044)$.

Furthermore, we also investigated the impact of expressive vocabulary on novel noun extension, as the language-groups were not matched on this measure. We conducted an ANCOVA with the group as a between-subjects factor, object category as a within-subjects factor, and expressive vocabulary as a covariate. The effect of the group was not significant, $F(1,27)=0.21, p=.64, \eta_{\mathrm{p}}^{2}=.003$. The effect of object category still reached significance $F(4,112)=18.1, p<.001, \eta_{\mathrm{p}}^{2}=.39$, so did the interaction, $F(4,112)=2.97, p=.022, \eta_{\mathrm{p}}{ }^{2}=.096$. Finally, the effect of the vocabulary did not reach significance, $F(1,27)=0.95, p=.34$, $\eta_{\mathrm{p}}^{2}=.015$.

Regarding chance level, language-matched children performed significantly above chance level for all categories of objects (all $p<.01$ ), except for the category of spatial configuration, $t(14)=1.88, p=.081$. Children with DLD performed above chance level for solid objects, animate objects, and functional role categories (all $p<.01$ ), but their performance was at chance level for non-solid substances, $t(14)=1.05, p=.31$, and spatial configuration categories, $t(14)=-0.84, p=.41$.

\section{Number of learning exemplars required for successful word extension}

\section{Age-matched groups}

To assess whether within-category comparison was beneficial to noun extension, we examined the number of learning exemplars each subject group needed to correctly extend novel nouns, and we then computed a mean for every object category. Recall that the number of learning exemplars was one in the first trial and two or three if children failed at the first and 
the second trial, respectively. If a child was successful identifying the correct object with one learning exemplar, it means that he or she did not need the comparison procedure. However, if he or she failed with one learning exemplar but succeeded with two or three, it means that a comparison was beneficial. We conducted a $2 \times 5$ ANOVA on the mean number of exemplars needed to correctly extend new words, with group (DLD vs. age-matched) as a betweensubjects factor and object category (solid objects vs. animate objects vs. non-solid substances vs. role categories vs. spatial configuration categories) as a within-subjects factor. The main effect of group did not reach significance, $F(1,28)=0.15, p=.70, \eta_{\mathrm{p}}^{2}=.005$. Because the Mauchly test indicated a violation of the sphericity assumption $(W=0.48, \mathrm{p}=.024)$, we corrected degrees of freedom using Greenhouse-Geisser corrections $(\varepsilon=0.75)$ for withinparticipant factors. The effect of object category reached significance, $F(3,84)=7.55, p<.001$, $\eta_{\mathrm{p}}^{2}=.21$. More interestingly, the interaction between the two factors, group and object category, was significant, $F(3,84)=5.85, p=.001, \eta_{\mathrm{p}}^{2}=.17$. This interaction indicated that while the number of exemplars differed as a function of object category in children with DLD, no such category-related differences were observed in age-matched children (all $p>.05$ ). As shown in Figure 4A, children with DLD relied on more learning exemplar in the spatial configuration category block than in the solid, animate (both $p<.001$ ), and functional role category blocks $(p=.028)$. Children with DLD also needed more exemplars for non-solid substances than for solid objects $(p=.003)$. Finally, children with DLD relied more on comparison than their agematched peers for non-solid substances $(p=.005)$ and spatial configuration categories $(p=$ .007). These results are presented in Figure 4A.

Figure 4

Mean number of learning exemplars needed for successful word extension as a function of the category of objects and as a function of the group (A. compares children with DLD and agematched TD children, B. compares children with DLD and language-matched TD children) 


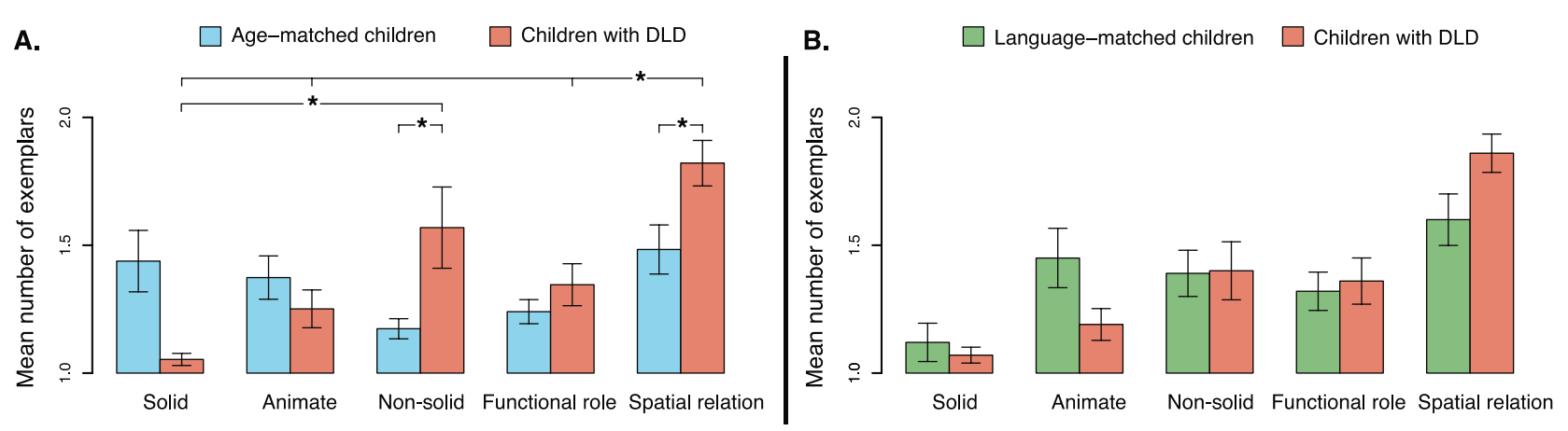

Note. Bars represent the standard error of the mean (SEM), $* p<.05$.

\section{Language-matched groups}

We conducted the same ANOVA on the mean number of exemplars required to correctly extend novel words with group (DLD vs. language-matched) and object category (solid objects vs. animate objects vs. non-solid substances vs. role categories vs. spatial configuration categories) as factors. The analysis did not yield a significant effect of group, $F(1,28)=.004$, $p=.95, \eta_{\mathrm{p}}^{2}<.001$ or a significant group by object category interaction, $F(4,112)=2.24$, $p=.069, \eta_{\mathrm{p}}^{2}=.074 .$. Moreover, the effect of object category was significant, $F(4,112)=18.0$, $p<.001, \eta_{\mathrm{p}}{ }^{2}=.39$. Post-hoc analyses using the Tukey method showed that while solid objects required less learning exemplars than the other object categories $(p=.047$ for animate objects, $\mathrm{p}=.002$ for non-solid substances, $p=.022$ for functional role categories, and $\mathrm{p}<.001$ for spatial configuration categories), spatial configuration categories required the most (all $p<.001$ ). These results are presented in Figure 4B.

\section{Response patterns to distractors' irrelevant features}

To examine whether children with DLD and their age-matched and language-matched peers exhibited different patterns of response bias towards irrelevant objects' features, we counted the number of times children picked each of the two distractor objects. When participants failed to choose the correct stimulus response (i.e., on trials with one, two or three learning exemplars), they selected the distractor whose shape or color (or another attribute) 
matched the target object's but was not relevant for category membership. Since solid and animate objects elicited a very low proportion of errors, we analyzed separately the data from non-solid substances, functional role categories, and spatial configuration categories only.

\section{Age-matched groups}

For non-solid substances, we conducted a $2 \times 2$ ANOVA with the distractor (shape vs. color) as a within-subject factor and the group (age-matched vs. DLD) as a between-subject factor on the number of responses. The effect of the group reached significance, $F(1,28)=7.58$, $p=.010, \eta_{\mathrm{p}}{ }^{2}=.21$, as children with DLD produced more incorrect responses than their agematched peers. The effect of the distractor was also significant, $F(1,28)=26.9, p<.001$, $\eta_{\mathrm{p}}{ }^{2}=.49$, with distractors based on shape being selected more than distractors based on color. Finally, the interaction also reached significance, $F(1,28)=4.74, p=.038, \eta_{\mathrm{p}}^{2}=.14$. Post-hoc analyses (Tukey HSD) revealed that children with DLD were more likely to select the shape distractor than their peers $(p=.048)$. They were also more likely to select the shape distractor than the color distractor $(p<.001$, see Table 5).

For functional role categories, we conducted a $2 \times 2$ ANOVA with the distractor (visual vs. thematic) as a within-subject factor and the group (age-matched vs. DLD) as a betweensubject factor on the number of responses. The effect of the group was significant, $F(1,28)=4.54, p=.042, \eta_{\mathrm{p}}^{2}=.14$, as children with DLD produced more incorrect responses than their peers. Neither the effect of the distractor, $F(1,28)=1.58, p=.22, \eta_{\mathrm{p}}{ }^{2}=.053$, nor the interaction, $F(1,28)=1.58, p=.22, \eta_{\mathrm{p}}^{2}=.053$, reached significance (see Table 5).

For spatial configuration categories, we conducted a $2 \times 2$ ANOVA with the distractor (shape vs. color) as a within-subject factor and the group (age-matched vs. DLD) as a betweensubject factor on the number of responses. The effect of the group reached significance, $F(1,28)=6.26, p=.018, \eta_{\mathrm{p}}{ }^{2}=.18$, as children with DLD produced more often incorrect responses than their peers. The effect of the distractor was also significant, $F(1,28)=50.4$, 
$p<.001, \eta_{\mathrm{p}}^{2}=.64$, with distractors based on the shape being selected more than distractors based on color. Finally, the most interesting result was the significant interaction, $F(1,28)=6.37, p=.017, \eta_{\mathrm{p}}^{2}=.18$. Post-hoc analyses (Tukey HSD) revealed that both groups selected significantly more shape distractors than color distractors, but this difference increases for children with DLD ( $p=.016$ for age-matched children and $p<.001$ for children with DLD).

\section{Table 5}

Number of incorrect responses as a function of the group (age-matched vs. DLD), distractor, and object class

Non-solid substances $\quad$ Functional role categories $\begin{gathered}\text { Spatial configuration } \\ \text { categories }\end{gathered}$

\begin{tabular}{lcccccc} 
& Shape & Color & Visual & Thematic & Shape & Color \\
\cline { 2 - 7 } & & & & & & \\
Children & & & & & & \\
$\begin{array}{l}\text { with } \\
\text { DLD } \\
(\mathrm{n}=15)\end{array}$ & $\begin{array}{c}6.80 \\
(4.87)\end{array}$ & $0.60(0.91)$ & $1.87(1.68)$ & $1.87(1.88)$ & $11.93(2.59)$ & $1.13(2.59)$ \\
$\begin{array}{l}\text { AM } \\
\text { children } \\
(\mathrm{n}=15)\end{array}$ & 2.60 & $0.07(0.26)$ & $0.53(0.83)$ & $1.40(1.35)$ & $6.07(1.33)$ & $0.93(1.33)$ \\
\hline
\end{tabular}

Note. $\mathrm{DLD}=$ developmental language disorder, $\mathrm{AM}=$ age-matched.

\section{Language-matched groups}

For non-solid substances, we conducted a $2 \times 2$ ANOVA with the distractor (shape vs. color) as a within-subject factor and the group (language-matched vs. DLD) as a betweensubject factor on the number of responses. The effect of the group was not significant, $F(1,28)=1.97, p=.17, \eta_{\mathrm{p}}^{2}=.066$, but the effect of the distractor was, $F(1,28)=24.9, p<$ $.001, \eta_{\mathrm{p}}{ }^{2}=.47$, as distractor based on shape were selected more than distractors based on color. Finally, the interaction did not reach significance, $F(1,28)=3.04, p=.092, \eta_{\mathrm{p}}{ }^{2}=.09($ see Table 6)

For functional role categories, we conducted a $2 \times 2$ ANOVA with the distractor (visual vs. thematic) as a within-subject factor and the group (language-matched vs. DLD) as a 
between-subject factor on the number of responses. The effect of the group did not reach significance, $F(1,28)=0.061, p=.81, \eta_{\mathrm{p}}^{2}=.003$, nor did the effect of the distractor, $F(1,28)=0.22, p=.64, \eta_{\mathrm{p}}{ }^{2}=.008$, or the interaction, $F(1,28)=0.097, p=.76, \eta_{\mathrm{p}}{ }^{2}=.003$.

For spatial configuration categories, we conducted a $2 \times 2$ ANOVA with the distractor (shape vs. color) as a within-subject factor and the group (language-matched vs. DLD) as a between-subject factor on the number of responses. The effect of the group was not significant, $F(1,28)=1.78, p=.19, \eta_{\mathrm{p}}{ }^{2}=.060$, but the effect of the distractor was, $F(1,28)=57.4, p<.001$, $\eta_{\mathrm{p}}{ }^{2}=.67$, with distractors based on shape being selected more than distractors based on color. Finally, the interaction did not reach significance, $F(1,28)=2.59, p=.12, \eta_{\mathrm{p}}{ }^{2}=.085$ (see Table $6)$.

Table 6

Number of incorrect responses as a function of the group (language-matched vs. DLD), distractor, and object class

\begin{tabular}{|c|c|c|c|c|c|c|}
\hline & \multicolumn{2}{|c|}{ Non-solid substances } & \multicolumn{2}{|c|}{ Functional role categories } & \multicolumn{2}{|c|}{$\begin{array}{c}\text { Spatial configuration } \\
\text { categories }\end{array}$} \\
\hline & Shape & Color & Visual & Thematic & Shape & Color \\
\hline $\begin{array}{l}\text { Children } \\
\text { with } \\
\text { DLD } \\
(\mathrm{n}=15)\end{array}$ & $\begin{array}{c}6.00 \\
(5.15)\end{array}$ & $0.47(0.92)$ & $1.87(1.51)$ & $1.93(1.94)$ & $13.60(7.59)$ & $0.47(0.83)$ \\
\hline $\begin{array}{l}\text { LM } \\
\text { children } \\
(\mathrm{n}=15)\end{array}$ & $\begin{array}{c}3.27 \\
(4.22)\end{array}$ & $0.60(0.91)$ & $1.87(2.00)$ & $2.20(2.04)$ & $9.33(8.13)$ & $0.80(1.47)$ \\
\hline
\end{tabular}

\section{Summary}

When one exemplar was provided, children with DLD performed worse than agematched children for non-solid substances, functional role categories, and spatial configuration categories. Children with DLD also performed better for solid and animate objects than for the other object classes. In contrast, 10-year-old (age-matched) TD children performed similarly 
for all object classes, while 8-year-old (language-matched) TD children performed better on trials with solid objects than with spatial configuration categories. Interestingly, receptive vocabulary had an impact on performance when children with DLD were matched with a same age group.

Concerning the impact of within-category comparison, children with DLD needed more opportunities to compare the stimuli to correctly generalize the novel words for spatial configuration categories than for the other object classes, and for non-solid substances than for solid objects. This result is important given that spatial categories are defined by relations and non-solid categories are not based on shape. Children with DLD also needed more exemplars than age-matched children to extend words of non-solid substances and spatial configuration categories. In a similar way, 8-year-old (language-matched) TD children required fewer exemplars for solid objects and more for spatial relation categories. In contrast, 10-year-old age-matched) TD children relied on the same number of exemplars for all the object classes.

Regarding response choices, children with DLD, as well as 8-year-old (languagematched) TD children, selected more the distractor based on shape than on color for non-solid substances and spatial configuration categories. In contrast, 10-year-old (age-matched) TD children selected more the distractor based on shape than on color for spatial configuration categories only.

\section{Discussion}

The overarching aim of this study was to evaluate the ability of children with DLD to extend nouns referring to diverse object classes. In addition, we explored the impact of lexicon size on new word extension and examined whether this process could be enhanced when children were given the opportunity to perform within-category comparisons. Finally, we analyzed the patterns of response biases when the participants failed to choose the correct object to extend novel words. The comparison between the conditions allowed us to better understand 
which object properties were used by our three groups when extending different kinds of nouns and to understand towards which properties children with DLD might be biased across lexical conditions.

\section{Higher-order generalization in children with DLD children}

Our results suggest that generalization abilities in children with DLD varied among different object classes. School-aged children with DLD performed similarly to TD children matched on age for solid and animate objects, but worse than their same-aged peers for nonsolid substances, functional role categories, and spatial configuration categories. Their sameage TD peers performed similarly and above chance level for all object classes. Ten-year-old TD children are able to focus on specific properties according to the object presented and are able to learn a property that is relevant to category membership with unfamiliar stimuli, as for spatial configuration categories.

Our results show for the first time that 10 -year-old children with DLD could perform similarly to same-aged peers and that they have developed a shape bias. Previously, Collisson et al. (2015) showed that 4-year-old preschool children with DLD were not able to rely on the shape bias. They also demonstrated weaknesses in detecting visual regularities, suggesting that children with DLD were not able to fully exploit visual information characterizing objects in their environment to support object word learning. Collisson et al. (2015) argued that successful emergence of the shape bias depends on the interplay of linguistic and visual information, and that both sources of information could be compromised in children with DLD. Studies that revealed difficulty in word extension in children with DLD involved preschool children (Gray, 1998; Schwartz et al., 1987). Our present results completed the picture: with age and experience, children with DLD could develop a shape bias, but this shape bias is developed later than in TD children. Linguistic and non-linguistic limitations in children with DLD could 
be a hindrance but not an obstacle: for concrete and frequent material, school-aged children with DLD reached the same performance levels as their peers. As solid objects are the most frequent in young children's daily life (Samuelson et al., 2008), children with DLD develop a shape bias, albeit some years later than their TD peers do. Kan and Windsor (2010) have also shown that the difference in word learning between children with DLD and their peers was greater in preschool- than school-age children. These authors suggested that less welldeveloped cognitive skills, such as attention, in young children with DLD (Kapa, Plante, \& Doubleday, 2017; Montgomery, 2008; Vissers, Koolen, Hermans, Scheper, \& Knoors, 2015) could impair their word learning abilities, while age-related factors, such as language or school experience could impact the performance of school-aged children. This may also be the case in our study: our school-aged participants with DLD may have compensated for their difficulty with age, experience and the maturation of their cognitive skills. However, our study also showed that children with DLD performed worse than their age-matched TD peers for nonsolid substances and relational nouns (i.e., functional role and spatial configuration categories). Children with DLD may have difficulty when they have to attend to properties other than shape. Interestingly, for non-solid substances and spatial relation categories, children with DLD performed at chance level. This may be explained by the fact that children with DLD require more time and experience with a specific object class to develop the appropriate attentional bias needed to extend it: school-aged children with DLD may not have enough experience with nonsolid substances and relational nouns to develop attentional biases for these object classes, especially as these biases appear later and are less robust than the shape bias in TD children (Samuelson et al., 2008).

In sum, children with DLD can extend novel nouns when the relevant property to category membership is a salient feature such as shape. However, they face difficulty when the property to be used involves relational information or texture. 


\section{Impact of the size of vocabulary on word extension}

By definition, children with DLD have language impairment. As previously mentioned, their vocabulary is characterized by limited breadth and depth (McGregor et al., 2013). Comparing word extension in children with DLD with typical children matched on vocabulary size is one way to understand the contribution of the vocabulary on word extension, in other words the contribution of a linguistic component on a generalization task. Based on a previous meta-analysis (Kan \& Windor, 2010), word learning was expected to be similar in children with DLD to children matched on lexical abilities. Results from the word extension task in younger TD children matched on a vocabulary measure showed that these 8-year-old children have good capacities for extending nouns of different kinds: they performed above chance level for every object class, except for spatial configuration categories. This may be because, for spatial configuration categories, children have to learn a property relevant for categorization which is arbitrary. It might also be stressed that the instruction format referred to the stimuli as an object whereas the relevant aspect was a non-salient spatial relation between two parts. We speculated that this unusual lexicalization situation might have triggered different learning processes. Children with DLD were at chance. The instructions might have pushed them toward shape rather than the relevant relation (see below).

As predicted, the performance of children with DLD was similar to their TD peers matched on vocabulary. Vocabulary and labeling experience have indeed been found to be related to word extension ability (Smith et al., 2002; Thom \& Sandhofer, 2009). Moreover, we found that receptive vocabulary influenced performance in the noun extension task for children with DLD and TD children matched on age. Our study therefore seems to confirm the relationship between word extension ability and vocabulary knowledge or labelling experience. Nevertheless, performance of children-with-DLD varied more as a function of object category compared with their TD language-matched peers. Moreover, children with DLD performed at 
chance level for non-solid substances, which was not the case for TD children matched on vocabulary. It seems that the generalization of substance names could discriminate between children with DLD and TD children of similar lexical abilities. This may be explained by the fact that textures are properties which are less salient than shape (Samuelson \& Horst, 2007; Samuelson et al., 2008). As children with DLD are impaired when detecting regularities in the visual domain (Collisson et al., 2015), texture could be a property especially difficult to identify as appropriate to generalization. Substance names also seem to be less frequent than solid object names, which are predominant in young children's daily life (Samuelson et al., 2008), and than relational categories, which are as frequent as object categories in adult discourse (Gentner, 2005). As labeling experience is crucial to word extension (Thom \& Sandhofer, 2009), a limited experience with substance names could make the extension of such categories challenging, especially for children with DLD.

\section{The benefit of within-category comparison}

Children rely on the comparison of within-category exemplars to extend new words. Comparison can be used to identify concrete features, such as shape or texture (Augier \& Thibaut, 2013; Smith et al., 2002), but it is especially useful for abstract words that rely on a conceptual or relational categorization criterion (Christie \& Gentner, 2010; Gentner et al., 2011). In contrast, DLD has been related to a difficulty in using comparisons (Leroy et al., 2012, 2014), while no studies, to our knowledge, have directly investigated the impact of comparison on novel word extension in children with DLD.

Regarding children with DLD, our results show that they relied on comparisons, as they needed more exemplars to extend spatial configuration categories and non-solid substances than TD children matched on age. Comparison is therefore beneficial for children with DLD, similarly as for TD children matched on vocabulary, even if some studies have shown difficulties in using comparison and structural alignment in DLD (Leroy et al., 2012, 2014). 
These findings are consistent with the study of Aguilar, Plante, and Sandoval (2018), which examined the role of object variability (one or three distinct exemplars) for new word learning in a between-group design comparing two groups of 5-year-old children with DLD. These authors found that children with DLD had better retention performance in a word extension task of solid objects when they had been presented with three distinct exemplars rather than one exemplar. The comparison of within-category exemplars seems to be beneficial for children with DLD when extending new words, for solid object names in preschool-aged children (Aguilar et al., 2018), and for spatial configuration categories and substance names in schoolaged children. Comparisons may be useful to these children for compensating for their difficulty with higher-order generalization. However, since school-aged children with DLD have developed a shape bias, they do not require comparison to extend solid object names.

The benefit of the comparison observed in children with DLD seems to work in the exact same way as in TD children. TD children matched on age did not require comparison to extend novel words, suggesting they have developed attentional biases which allow them to extend novel words following a single learning exemplar. In contrast, language-matched TD children used the comparison like children with DLD. These language-matched TD children needed fewer exemplars for solid objects and more for spatial configuration categories, compared with the other object classes. This could mean that the shape bias is fully developed in these younger children, while they still rely on comparison when they have to learn the relevant property to category membership for nouns other than object names, as for spatial configuration categories.

\section{The impact of shape on generalization}

The analysis of incorrect responses has generated a better understanding of the strategies used by children with DLD. These children produced more incorrect responses than TD children matched on age for non-solid substances, functional role categories, and spatial configuration categories when one, two, or three learning exemplars were provided. 
Interestingly, children with DLD selected the distractor based on shape to a larger extent for non-solid substances and spatial configuration categories. This focus on shape suggests a less flexible use of dimensions other than shape when they are conceptually relevant for generalization. This is consistent with other studies showing that children with DLD were more dependent on perceptual features than their age-matched peers and that they required the support of perceptual features to identify relational similarities in linguistic or non-linguistic reasoning tasks (Leroy et al., 2012, 2014). The present study suggests that children with DLD are biased towards shape even when shape is not relevant, which prevents them from extending nouns for which texture or a relational property has to be used. Several interpretations of this bias may be distinguished.

First, children with DLD may overgeneralize the shape bias. These children indeed have difficulty detecting regularities in the visual domain and identifying contingencies between object properties and categories (Collisson et al., 2015). It is therefore likely that, once they identified shape as a relevant property to extend novel words, they use it indistinguishably for diverse object classes. Moreover, children with DLD are impaired when they have to identify relational similarities (Leroy et al., 2012, 2014): they may have difficulty identifying a novel relational criterion to extend spatial configuration categories and rely on shape, which is easily detectable.

Second, they may have more difficulty inhibiting perceptual information such as shape. Inhibition has been related to the ability to move beyond perceptual matches in new word extension tasks. Snape and Krott (2018) found a positive relationship between the ability to extend new words on a conceptual rather than perceptual feature and inhibition as measured by a classical inhibition task in preschool-aged TD children. This relation was regardless of age and general cognitive maturation. Nevertheless, DLD has been linked to an executive function deficit, especially for inhibition (Cuperus, Vugs, Scheper, \& Hendriks, 2014; Im-Bolter, 
Johnson, \& Pascual-Leone, 2006). It is therefore possible that poor inhibition abilities lead to an overwhelming focus on salient perceptual features (such as shape) and prevent children with DLD from using texture or relational features to generalize novel nouns referring to non-solid substances or spatial relations. Future studies will be needed to further investigate this hypothesis by evaluating inhibition in children with DLD and in TD children during word extension tasks.

Third, no differences appeared between children with DLD and TD children matched on vocabulary, as these TD children also selected the distractor based on shape more than the distractor based on color for non-solid substances and spatial configuration categories. This overwhelming bias towards shape may therefore be related to lexical abilities. Word extension has already been linked to vocabulary and labeling experience (Smith et al., 2002; Thom \& Sandhofer, 2009). In this study also, we saw that word extension ability was similar between children with DLD and TD children when vocabulary was controlled, while vocabulary impacted word extension ability when children with DLD and TD children were matched on age. It is therefore possible that children with DLD and their language-matched peers have had more experience with solid object names, which drives them to focus on shape preferentially. It is also likely that the impact of vocabulary differs according to a child's age: it may be significant for younger children, but may diminish with language experience and the development of attentional biases, such as the shape bias for solid object names.

Another possibility would be that non-solid objects were not perfectly identified these items as non-solid (or some of them) but rather as stimuli with a shape. Indeed, from a methodological point of view, it is virtually impossible to remove all the shape cues from visual stimuli. However, both TD groups chose texture matches beyond chance which was not the case for children with DLD. This strongly suggests that despite some potential heterogeneity between stimuli in terms of shape affordance, the TD children went for the texture choice in 
this condition which, also, did not differ significantly from the animate condition (for example) whereas children with DLD chose significantly less the relevant dimension in the non-solid dimension than in the animate condition. Taken together, these results suggest that non-solid objects were uniformly perceived as solid objects and remained special for children with DLD.

\section{Implications}

Our results provide new evidence about lexical deficits in children with DLD. There is indeed considerable debate regarding lexical capacities in these children: some authors consider these abilities unimpaired (Ullman \& Pierpont, 2005), while others identified weaknesses in lexical learning and semantic representation (Kan \& Windsor, 2010; McGregor et al., 2013). Our data suggest that a crucial step of word learning, namely word extension, is impaired in DLD. It is likely that children with DLD have difficulty detecting regularities between language (i.e. object names) and the visual domain (i.e. object categories), which may explain the fact that the shape bias is still not observed in preschool children with DLD (Collisson et al., 2015; Kucker et al., 2019). Our results suggest that once this principle is established, children with DLD use it more rigidly for most noun categories. Moreover, word extension ability in children with DLD bears some resemblance to that of their language-matched peers. This has already been shown for word learning (Kan \& Windsor, 2010). This also reinforces the relationship observed between vocabulary and word extension ability in TD children (Smith et al., 2002; Thom \& Sandhofer, 2009). Our results are also compatible with a theoretical framework on DLD of a domain general implicit learning deficit. Finally, this study also raises insights into how the procedure used to evaluate lexicon in children with DLD can impact their performance: first, assessing word learning, word knowledge or word extension triggers diverse competencies and will lead to different results (Collisson et al., 2015; Kan \& Windsor, 2010; McGregor et al., 2013). Second, the object categories used will also impact the evaluation: using solid object names only, especially if children are school-aged, may lead to an inaccurate picture of their 
lexical capacities. In this case, it is possible that no deficit will be visible while difficulties would emerge if relational nouns or non-solid objects had been tested. Our study suggests that word extension tasks on abstract lexicon such as non-solid or relational categories were discriminant between school-age children with or without DLD. Additional studies are needed to investigate the usefulness of these tasks for clinical assessment purposes.

\section{Limitations}

This study has some limitations that need to be addressed. First, the sample was relatively small, and a higher number of participants could have increased statistical power. Second, we chose to present two-dimensional objects and not real objects, and this could also have influenced the results, mainly for non-solid substances. Some authors indeed found that this variable had an impact on the shape bias in preschool-aged TD children: shape was used more often with two-dimensional than with three-dimensional inanimate objects in a word extension task (Davidson, Rainey, Vanegas, \& Hivert, 2018). Moreover, three-dimensional objects were used in previous studies of word extension using substance names (Soja et al., 1991), while we used two-dimensional objects, which could have impacted our results. Additional information that we should have thoroughly collected is an estimation of children's vocabulary. We found that vocabulary was related to noun extension, but our vocabulary measures did not allow us to evaluate the lexicon size for different object classes. It is indeed possible that the ability to extend names for non-solid substances would be related to the number of non-solid substances that a child can name. This is consistent with the study of Thom and Sandhofer (2009), who found that the number of color names that children learned was related to their ability to extend novel color names. However, to our knowledge, there is no standardized measure of vocabulary allowing for this kind of evaluation. Finally, word extension ability may have been related to inhibition. As has already been discussed, inhibition has been linked to word extension for nouns whose category membership relies on conceptual 
features (Snape \& Krott, 2018). As children with DLD usually have poor inhibition capacities (Im-Bolter et al., 2006), this would have been an interesting hypothesis to investigate.

\section{Conclusions}

This study aimed at evaluating word extension in school-aged children with DLD, using various object classes. Our objective was to examine children's ability to identify regularities between object properties and category organization, and so to select the relevant feature to category membership according to the object presented. Results revealed that children with DLD were impaired compared to TD children matched by age when extending non-solid substances, functional role, and spatial configuration categories, while their performance approached the performance of TD children matched on language. Comparisons were beneficial for children with DLD, especially for spatial configuration categories. However, these children were highly influenced by shape, even if it was not a relevant property to category membership. Ten-year-old TD children (matched on age) were successful in extending words for all the object classes presented. Eight-year-old TD children (matched on language) also had good capacities, except for spatial configuration categories, for which a categorization criterion had to be learned. In this case, these children relied on the comparison of within-category exemplars to extend new words. Our study presents new evidence about noun extension in children with DLD and in TD children. To our knowledge, this is the first study that compares word extension ability for five different kinds of entity names in TD children and in children with language disorders. Our work contributes some interesting data about word extension in children with DLD, especially regarding the benefit of comparison and the impact of salient features such as shape. Moreover, our findings reinforce the idea that children are able to learn regularities between object properties and category organization, and so to focus on diverse features according to the object presented when extending new nouns. Finally, this study confirms the beneficial role of comparison on new word extension. 


\section{Acknowledgments}

We thank the teachers, speech therapists, parents, and children who participated in the project.

\section{Funding}

This work was supported by the FRESH fund of the F.R.S.-FNRS.

\section{Declarations of interest: none}

\section{References}

Aguilar, J. M., Plante, E., \& Sandoval, M. (2018). Exemplar variability facilitates retention of word learning by children with specific language impairment. Language, Speech, and Hearing Services in Schools, 49(1), 72-84.

Alt, M., Plante, E., \& Creusere, M. (2004). Semantic features in fast-mapping. Journal of Speech, Language, and Hearing Research, 47(2), 407-420.

Augier, L., \& Thibaut, J.P. (2013). The benefits and costs of comparisons in a novel object categorization task: Interactions with development. Psychonomic Bulletin \& Review, 20(6), 1126-1132.

Bishop, D. V., Snowling, M. J., Thompson, P. A., Greenhalgh, T., \& Catalise-2 Consortium. (2017). Phase 2 of CATALISE: A multinational and multidisciplinary Delphi consensus study of problems with language development: Terminology. Journal of Child Psychology and Psychiatry, 58(10), 1068-1080.

Bloom, P. (2002). How children learn the meanings of words. Cambridge: MIT Press.

Christie, S., \& Gentner, D. (2010). Where hypotheses come from: Learning new relations by structural alignment. Journal of Cognition and Development, 11(3), 356-373.

Collisson, B. A., Grela, B., Spaulding, T., Rueckl, J. G., \& Magnuson, J. S. (2015). Individual differences in the shape bias in preschool children with specific language impairment and 
typical language development: Theoretical and clinical implications. Developmental Science, 3, 373-388.

Cuperus, J., Vugs, B., Scheper, A., \& Hendriks, M. (2014). Executive function behaviours in children with specific language impairment (SLI). International Journal of Developmental Disabilities, 60(3), 132-143.

Davidson, D., Rainey, V. R., Vanegas, S. B., \& Hilvert, E. (2018). The effects of type of instruction, animacy cues, and dimensionality of objects on the shape bias in 3-to 6-yearold children. Infant and Child Development, 27, e2044.

Dunn, L. M., Theriault-Whalen, C. M., \& Dunn, L. M. (1993). Echelle de vocabulaire en images peabody. Toronto: Psychan.

Gentner, D. (2005). The development of relational category knowledge. In L. Gershkoff-Stowe \& D. H. Rakison, (Eds.), Building object categories in developmental time (pp. 245-275). Hillsdale: Lawrence Erlbaum Associates.

Gentner, D., Anggoro, F. K., \& Klibanoff, R. S. (2011). Structure mapping and relational language support children's learning of relational categories. Child Development, 82(4), 1173-1188.

Gentner, D., \& Namy, L. L. (1999). Comparison in the development of categories. Cognitive Development, 14, 487-513.

Gershkoff-Stowe, L., \& Smith, L. B. (2004). Shape and the first hundred nouns. Child Development, 75(4), 1098-1114.

Gray, S. (1998). Lexical acquisition by children with specific language impairment: Phonological and semantic effects on and performance predictions among fast mapping, word learning, and word extension (Doctoral dissertation). University of Arizona, Tucson, USA. 
Imai, M., \& Gentner, D. (1997). A cross-linguistic study of early word meaning: Universal ontology and linguistic influence. Cognition, 62(2), 169-200.

Im-Bolter, N., Johnson, J., \& Pascual-Leone, J. (2006). Processing limitations in children with specific language impairment: The role of executive function. Child Development, 77(6), $1822-1841$

Jackson, E., Leitao, S., \& Claessen, M. (2016). The relationship between phonological shortterm memory, receptive vocabulary, and fast mapping in children with specific language impairment. International Journal of Language \& Communication Disorders, 51(1), 6173.

Jones, S.S., \& Smith, L.B. (1998). How children name objects with shoes. Cognitive Development, 13, 323-334.

Jones, S.S., Smith, L. B., \& Landau, B. (1991). Object properties and knowledge in early lexical learning. Child Development, 62, 499-516.

Kan, P.F., \& Windsor, J. (2010). Word learning in children with primary language impairment: A meta-analysis. Journal of Speech, Language, and Hearing Research, 53, 739-756.

Keil, F. C., \& Batterman, N. (1984). A characteristic-to-defining shift in the development of word meaning. Journal of Verbal Learning and Verbal Behavior, 23(2), 221-236.

Khomsi, A. (2001). ELO: Evaluation du langage oral. Paris: ECPS.

Kucker, S. C., Samuelson, L. K., Perry, L. K., Yoshida, H., Colunga, E., Lorenz, M. G., \& Smith, L. B. (2019). Reproducibility and a unifying explanation: Lessons from the shape bias. Infant Behavior and Development, 54, 156-165.

Lawrence, MA. (2011). ez: Easy analysis and visualization offactorial experiments. R package version 3.0-0.

Lecocq, P. (1996). L’ECOSSE: Une épreuve de compréhension syntaxico-sémantique. Villeneuve-d'Ascq: Presses Universitaires du Septentrion. 
Leonard, L. B. (2014). Children with specific language impairment. Cambridge: The MIT Press.

Leroy, S., Maillart, C., \& Parisse, C. (2014). Analogical mapping across modalities in children with specific language impairment (SLI). Research in Developmental Disabilities, 35(9), $2158-2171$.

Leroy, S., Parisse, C., \& Maillart, C. (2012). Analogical reasoning in children with specific language impairment. Clinical Linguistics and Phonetics, 26(4), 380-395.

Lété, B., Sprenger-Charolles, L., \& Colé, P. (2004). Manulex: A grade-level lexical database from French elementary-school readers. Behavior Research Methods, Instruments, \& Computers, 36, 156-166.

Mathôt, S., Schreij, D., \& Theeuwes, J. (2012). OpenSesame: An open-source, graphical experiment builder for the social sciences. Behavior Research Methods, 44(2), 314-324.

McGregor, K. K., Oleson, J., Bahnsen, A., \& Duff, D. (2013). Children with developmental language impairment have vocabulary deficits characterized by limited breadth and depth. International Journal of Language \& Communication Disorders, 48(3), 307-319.

Montgomery, J. W. (2008). Role of auditory attention in the real-time processing of simple grammar by children with specific language impairment: A preliminary investigation. International Journal of Language \& Communication Disorders, 43(5), 499-527.

Namy, L. L., \& Gentner, D. (2002). Making a silk purse out of two sow's ears: Young children's use of comparison in category learning. Journal of Experimental Psychology: General, 131(1), 5-15.

Nation, I. S. (2013). Teaching \& learning vocabulary. Boston: Heinle Cengage Learning.

Pandolfe, J. M., Wittke, K. and Spaulding, T. J. (2016). Do adolescents with specific language impairment understand driving terminology? Language, Speech, and Hearing Services in Schools, 47, 324-333. 
Perry, L. K., Samuelson, L. K., \& Burdinie, J. B. (2014). Highchair philosophers: The impact of seating context-dependent exploration on children's naming biases. Developmental Science, 17(5), 757-765.

Perry, L. K., Samuelson, L. K., Malloy, L. M., \& Schiffer, R. N. (2010). Learn locally, think globally: Exemplar variability supports higher-order generalization and word learning. Psychological Science, 21(12), 1894-1902.

Samuelson, L. K., \& Horst, J. S. (2007). Dynamic noun generalization: Moment-to-moment interactions shape children's naming biases. Infancy, 11(1), 97-110.

Samuelson, L. K., Horst, J. S., Schutte, A. R., \& Dobbertin, B. N. (2008). Rigid thinking about deformables: Do children sometimes overgeneralize the shape bias? Journal of Child Language, 35(3), 559-589.

Samuelson, L. K., \& Smith, L. B. (2000). Children's attention to rigid and deformable shape in naming and non-naming tasks. Child Development, 71(6), 1555-1570.

Schwartz, R. G., Leonard, L. B., Messick, C., \& Chapman, K. (1987). The acquisition of object names in children with specific language impairment: Action context and word extension. Applied Psycholinguistics, 8(3), 233-244.

Smith, L., Jones, S., Landau, B., Gershkoff-Stowe, L., \& Samuelson, L. (2002). Object name learning provides on-the-job training for attention. Psychological Science, 13(1), 13-19.

Snape, S., \& Krott, A. (2018). The role of inhibition in moving beyond perceptually focused noun extensions. First Language, 38(1), 95-108.

Soja, N.N., Carey, S., \& Spelke, E.S. (1991). Ontological categories guide young children's inductions of word meaning: Object terms and substance terms. Cognition, 38, 179-211.

Thibaut, J.-P., \& Witt, A. (2015). Young children's learning of relational categories: Multiple comparisons and their cognitive constraints. Frontiers in Psychology, 6, 643. 
Thibaut, J.-P., \& Witt, A. (2017). Generalizing novel names in comparison settings: Role of conceptual distance during learning and at test. In Gunzelman, G., Howes, A., Tenbrink, T., \& Davelaar, E. (Eds.), Proceedings of the 39th Annual Meeting of the Cognitive Science Society (pp. 3314-3319). Austin: Cognitive Science.

Thom, E. E., \& Sandhofer, C. M. (2009). More is more: The relationship between vocabulary size and word extension. Journal of Experimental Child Psychology, 104, 466-473.

Trauner, D., Wulfeck, B., Tallal, P., \& Hesselink, J. (2000). Neurological and MRI profiles of children with developmental language impairment. Developmental Medicine and Child Neurology, 42, 470-475

Ullman, M. T., \& Pierpont, E. I. (2005). Specific language impairment is not specific to language: The procedural deficit hypothesis. Cortex, 41(3), 399-433.

Wechsler, D., \& Naglieri, J. (2009). Echelle non verbale d'intelligence de Wechsler. Montreuil: ECPA.

Windfuhr, K. L., Faragher, B., \& Conti-Ramsden, G. (2002). Lexical learning skills in young children with specific language impairment (SLI). International Journal of Language \& Communication Disorders, 37(4), 415-432. 


\section{APPENDIX}

Non-words used in the experimental task

\begin{tabular}{|c|c|c|c|c|c|}
\hline /bate/ & /bofe/ & /bodã/ & /buni/ & /buse/ & / Save/ \\
\hline /koRi/ & /kudã/ & /deto/ & /dysi/ & /dyfã// & /dyzõ/ \\
\hline /fasi/ & /falãa/ & /fave/ & /line/ & /lito/ & $/ \mathrm{mal} \tilde{\mathrm{o}} /$ \\
\hline /mapi/ & /mise/ & /padi/ & /patã/ & /scto/ & /sudi/ \\
\hline /suve/ & $/ \operatorname{tav} \tilde{\mathbf{a}} /$ & /tysã / & /vido/ & /vize/ & /vile/ \\
\hline
\end{tabular}

